\title{
The pre-ZAMS nature of Mol160/IRAS 23385+6053 confirmed by Spitzer
}

\author{
S. Molinari ${ }^{1}$, F. Faustini ${ }^{1}$, L. Testi ${ }^{2,3}$, S. Pezzuto ${ }^{1}$, R. Cesaroni ${ }^{2}$, and J. Brand ${ }^{4}$ \\ 1 Istituto Fisica Spazio Interplanetario - INAF, via Fosso del Cavaliere 100, 00133 Roma, Italy \\ e-mail: [molinari; faustini;pezzuto]@ifsi-roma.inaf.it \\ 2 Osservatorio Astrofisico di Arcetri - INAF, Largo E. Fermi 5, 50125 Firenze, Italy \\ e-mail: [lt; cesa]@arcetri.astro.it \\ 3 ESO, Karl-Schwarzschild-Strasse 2, 85748 Garching, Germany \\ e-mail: 1testi@eso.org \\ 4 Istituto di Radioastronomia - INAF, via Gobetti 101, 40129 Bologna, Italy \\ e-mail: brand@ira.inaf.it
}

Received 20 March 2008 / Accepted 4 June 2008

\begin{abstract}
Context. The formation of massive stars goes through phases that remain heavily obscured until the object is well along on the main sequence. The identification of massive YSOs in different evolutionary phases is therefore particularly difficult, and requires a statistical approach with large samples of candidate objects to determine the observational signatures of these different phases. Aims. A mandatory activity in this context is the identification and characterisation of all the phases that a massive forming YSO undergoes. It is of particular interest to verify the observability of the phase in which the object is rapidly accreting while not yet igniting the fusion of hydrogen that marks the arrival on the ZAMS.

Methods. One of the candidate prototypical objects for this phase is Mol160/IRAS 23385+6053, which has been the subject of detailed studies that confirmed and strengthened the possibility that this massive YSO may be in a pre-hot core stage. We further investigate this issue by means of Spitzer imaging and spectroscopy in the 5-70 $\mu \mathrm{m}$ range.

Results. The dense core of Mol160/IRAS 23385+6053, which up to now had only been detected at submillimeter and millimeter wavelengths, with only upper limits below $20 \mu \mathrm{m}$, has been revealed for the first time at 24 and $70 \mu \mathrm{m}$ by Spitzer. These observations confirm the earlier assumptions that this object is dominant at far-IR wavelengths. The complete $24 \mu \mathrm{m}-3.4 \mathrm{~mm}$ continuum cannot be fitted with a standard model of a zero-age main-sequence (ZAMS) star embedded in an envelope. A simple greybody fit yields a mass of $220 M_{\odot}$. The luminosity is slightly in excess of $3000 L_{\odot}$, which is a factor of 5 less than previous estimates when only IRAS fluxes were available between 20 and $100 \mu \mathrm{m}$. The source is under-luminous by the same factor with respect to UCHII regions or hot-cores of similar circumstellar mass, and simple models show that this is compatible with an earlier evolutionary stage. Spectroscopy between 5-40 $\mu \mathrm{m}$ shows that the physical conditions are typical of a photo-dissociated or photo-ionized region. The required UV illumination can be provided by some of the other sources revealed at $\lambda \leq 24 \mu \mathrm{m}$ in the same star-forming region, that can be plausibly modeled as moderately embedded intermediate-mass ZAMS stars.

Conclusions. Our results strengthen the suggestion that the central core in Mol160/IRAS 23385+6053 is a massive YSO actively accreting from its circumstellar envelope and that it has not yet begun hydrogen fusion.
\end{abstract}

Key words. stars: formation - stars: pre-main sequence - infrared: ISM

\section{Introduction}

The identification and the characterisation of all the different phases that an intermediate and high mass forming object undergoes during its approach toward the main sequence (MS) has received increasing attention in recent years. Besides its importance to complete our understanding of the star formation process in general, this research has interesting consequences in a broader context. For example, the number of OB ZAMS stars indirectly estimated using a variety of tracers like the radio continuum or the $\mathrm{H} \alpha$ emission, is widely used to estimate the star formation rate and -efficiency, critical parameters to measure the history of star formation in our Galaxy as well as in external galaxies (McKee \& Williams 1997; Kennicutt 1998).

The identification of a "protostar" for intermediate and highmass objects is more difficult than for those of low-mass, because massive objects reach the ZAMS while still accreting material. Up to that moment they remain deeply embedded in their natal molecular cloud, and unlike their lower mass counterparts they lack a visible pre-MS phase. With accretion rates lower than $10^{-5} M_{\odot} \mathrm{yr}^{-1}$ the existence of this classical pre-MS phase is limited to objects of mass $M \leq 8 M_{\odot}$. Accretion, however, plays a very important role raising this mass limit and allowing the arrival on the ZAMS at higher stellar masses if accretion proceeds at much higher rates; the Young Stellar Object (YSO) joins the ZAMS at $M=16 M_{\odot}$ for $\dot{M}=10^{-4} M_{\odot} \mathrm{yr}^{-1}$ (Palla \& Stahler 1992), and well beyond $20 M_{\odot}$ for even higher rates. Such rates indeed seem plausible, given the mass loss rates measured from outflows emanating from massive YSOs (Zhang et al. 2001; Beuther et al. 2002), and are also predicted by theoretical models (McKee \& Tan 2003) where accretion rates increase with the mass of the forming core.

We recently completed a preliminary study of the Spectral Energy Distributions (SEDs) of a sample of massive YSOs in various evolutionary stages (Molinari et al. 2008) which 
suggests that objects more massive than $8 M_{\odot}$ may indeed be observable in a pre-ZAMS phase as pure far-infrared (undetected below $20 \mu \mathrm{m}$ ) objects underluminous with respect to Hot-Cores or Ultra Compact (UC) HII regions for a given envelope mass. Mol160/IRAS 23385+6053 clearly manifests its difference in multiwavelength appearance with respect to known Hot-Cores or UCHII regions, and is perhaps a template for this class of massive analogues of classical Class 0 objects (Molinari et al. 1998b). The strong millimetric peak, which appears to harbor the driving source of a molecular outflow, is anti-correlated with the complex emission morphology visible at mid-IR wavelengths (Molinari et al. 1998b) and with the extended radio continuum emission found in the region (Molinari et al. 2002). Detailed spectroscopic investigation confirmed that from a chemistry viewpoint, the millimeter core of Mol160/IRAS 23385+6053 appears to be on the verge of turning into a Hot-Core (Thompson $\&$ Macdonald 2003) and, with $T \leq 50 \mathrm{~K}$ (Fontani et al. 2004), is considerably colder than typical Hot-Cores (HC) of similar envelope mass.

The suggestion that bolometric luminosity and SED morphology may be promising evolutionary indicators is based on the coupled analysis of mid-IR and submillimetric data. For the study of large samples in these wavelength ranges, where survey data are available, the accessible spatial resolutions of $10-20^{\prime \prime}$ are not sufficient to pinpoint each and every emitter in the clustered environments where massive YSOs are found, but are enough to recognize systematic differences between classes of objects. Similar resolutions in the far-infrared, where they are most crucial since it is where the SEDs peak, will not be accessible until the Herschel satellite is available; in the meantime, an important confirmation of the scenario proposed by Molinari et al. (2008) may be provided by the Spitzer satellite. Stecklum et al. (2005) presented MIPS continuum data of Mol160/IRAS 23385+6053 and concluded that this source was not a high-mass protostar. We here reconsider the same MIPS data and provide an alternative and more plausible intepretation which is more consistent with the independent evidence of the pre-HC nature of this object and the more general picture of the luminosity evolution of massive YSOs. Additional Spitzer IRS spectroscopy is also presented, and the implications for the star formation activity of the entire region are discussed.

\section{Observations and data a nalysis}

Data for source Mol160/IRAS 23385+6053 were acquired with the Spitzer satellite in two Guaranteed Time programs. The first program used the MIPS instrument (Rieke et al. 2004) to acquire photometric images at 24 and $70 \mu \mathrm{m}$. The $128 \times 128 \mathrm{Si}$ :As array at $24 \mu \mathrm{m}$ has a pixel size of 2 '. $49 \times 2$ '. 60 and a field-ofview (FOV) of 5.4 $\times 5.4$, while the $70 \mu \mathrm{m}$ array consists of $32 \times 32$ Ge:Ga matrix with a pixel size of $5^{\prime \prime} .24 \times 5$ ". 33 and a FOV of $2 ! 7 \times 1$ '.35. The data were taken with an exposure time of $3 \mathrm{~s}$, and were repeated twice at $24 \mu \mathrm{m}$ and 3 times at $70 \mu \mathrm{m}$. One cycle of the basic $24 \mu \mathrm{m}$ photometry observation consisted of 14 offset images of the field combined with classical techniques of chopping (along-scan mirror motion) and dithering. One cycle of $70 \mu \mathrm{m}$ observations produces, with the same method, 12 images of the field. Also an estimate of background-foreground contribution is made acquiring an image off-source with chopping technique.

The second program contains spectroscopy mapping with the IRS instrument (Houck et al. 2004) using three of the available modules. The "Long High" (LH) module covers a range between 19 and $37 \mu \mathrm{m}$ at a spectral resolution of $R=600$ with a FOV of 22 '. $3 \times 11^{\prime \prime} .1$ and a pixel size of 4.'5. The "Short High" (SH) module covers with similar spectral resolution the range between 10 and $19.5 \mu \mathrm{m}$ with a FOV of $11^{\prime \prime} .3 \times 4$.' 7 and a pixel size of 2'.3. The "Short Low" (SL) slits were used to cover the range between 5.6 and $14.5 \mu \mathrm{m}$. The SL module is composed of two sub-modules at different wavelengths; one covers the range between 7.4 and $14.5 \mu \mathrm{m}$ at $R=16$, with a FOV of $57^{\prime \prime} \times 3$.' 7 and a pixel size of 1 ." 8 , and the second covers at $R=8$ a range between 5.2 and $8.7 \mu \mathrm{m}$, with a FOV of $57^{\prime \prime} \times 33^{\prime \prime} 6$ and a pixel size of 1".8. Observations consisted of one integration with a ramp duration of $6 \mathrm{~s}$. The area around the position of the millimeter emission peak was covered with a small $3 \times 2$ map.

For both programs we downloaded the Basic Calibrated Data products from the Spitzer archive. Pipeline versions used for automatic processing were S.11.0.2 for MIPS data, and S15.3.0 for IRS.

\subsection{Source photometry: near-IR to millimeter}

Figure 1a shows the Mol160/IRAS 23385+6053 field at $24 \mu \mathrm{m}$ (greyscale image) and at $70 \mu \mathrm{m}$ (contours). The extraction and photometry of point sources from the $24 \mu \mathrm{m}$ image was attempted using several publicly available packages for automatic source detection and photometry (SExtractor, IRAF, MOPEX). As is apparent from the image, the various peaks that are visible sit on a complex patch of extended emission which is strongly variable on small and large scales. This poses severe problems for reliable source detection and photometry since the various packages we tried assume either a constant background, or estimate one by using a box of fixed size across the image. Sources like A in the figure, for example, are undetected with SExtractor and also with MOPEX if the box for estimating the background is too large. Using a small box would solve this, but on the other hand leads one to significantly understimate the fluxes for sources like $\mathrm{N}$ or $\mathrm{E}$ which instead lie on a less variable and broader patch of extended emission; discrepancies in the fluxes of up to a factor of 5 are found for some objects using different packages. For this reason we decided to visually identify compact peaks in the map, and to manually perform the photometry at $24 \mu \mathrm{m}$ with custom procedures using the GILDAS package, optimising by hand the area for the background estimate.

At $70 \mu \mathrm{m}$ the situation is less complex because due to the lower spatial resolution the various sources in the area are blended in a large patch of emission; among the sources detected at $24 \mu \mathrm{m}$ source $\mathrm{A}$ is the one that more clearly stands out above this plateau (Table 1). A slight offset is apparent between the position of the 24 and $70 \mu \mathrm{m}$ peaks; we will comment on this below. The size of this peak was estimated analyzing cross-cut brightness profiles; this information was transferred to the AIPS task JMFIT to fit a composite 2D-Gaussian and a plateau resulting in an integrated flux density of $65 \mathrm{Jy}$ for the compact central peak coincident with source A. The integrated intensity of the entire $70 \mu \mathrm{m}$ emission structure, after subtraction of a constant emission level clearly detectable above the noise at the borders of the image, is $\sim 350 \div 400 \mathrm{Jy}$, compatible with the $60 \mu \mathrm{m}$ IRAS flux density. The $70 \mu \mathrm{m}$ flux density that can be assigned to the central massive core coincident with source A is then much lower than the $60 \mu \mathrm{m}$ IRAS flux density, contrary to the assumptions made by Fontani et al. (2004). In that work we carried out a farIR extrapolation of the mid-IR emission of the bright patches revealed in the ISOCAM images (Molinari et al. 1998b), which are also visible in the present $24 \mu \mathrm{m}$ image, and concluded that most of the IRAS 60 and $100 \mu \mathrm{m}$ flux densities could be assigned to the central core. The MIPS observations, however, provide a 

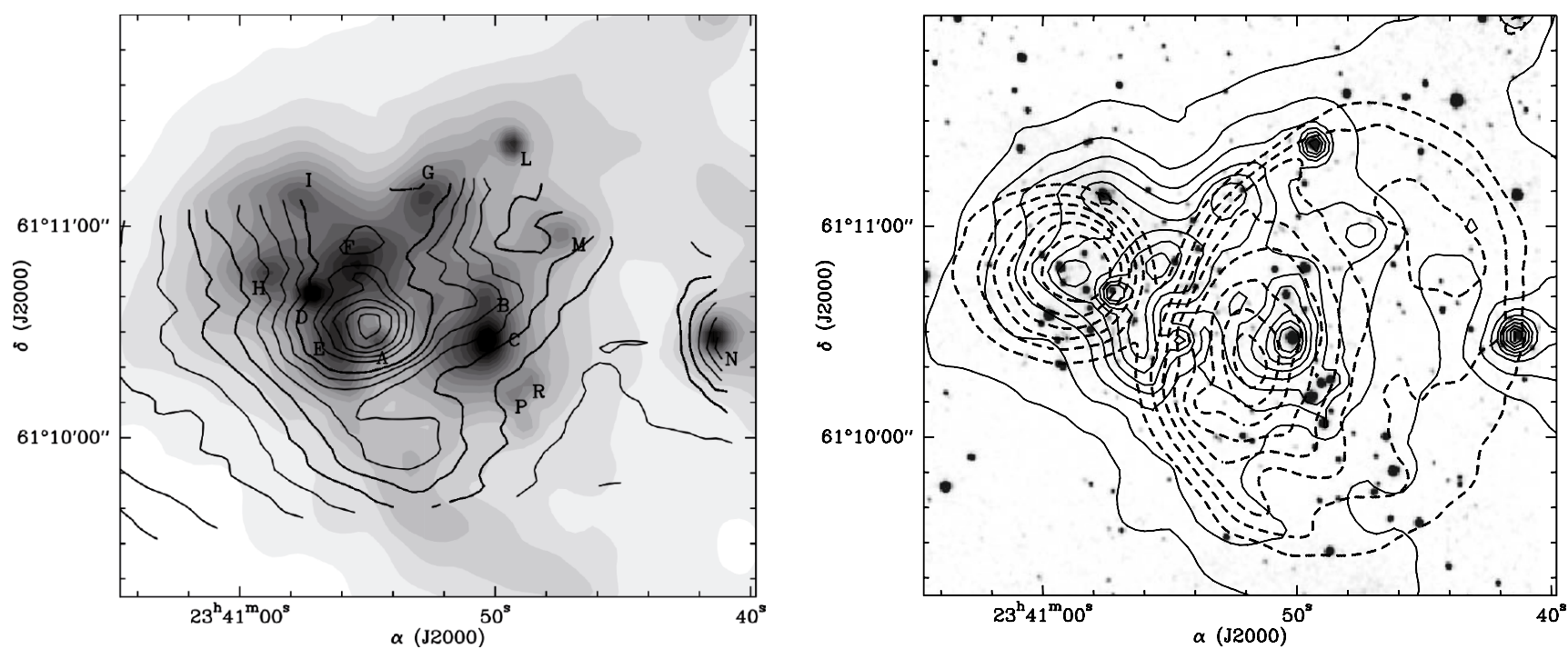

Fig. 1. a) (Left) MIPS $24 \mu \mathrm{m}$ grey-scale image of the Mol160/IRAS 23385+6053 field, with superimposed the contours of the $70 \mu \mathrm{m}$ emission; the letters mark the identified sources. b) (Right) $2.2 \mu \mathrm{m}$ image of the same region, at approximately the same scale; solid countours represent the $24 \mu \mathrm{m}$ continuum emission, while the dashed contours show the $3.6 \mathrm{~cm}$ thermal free-free emission.

Table 1. Photometry of sources in the field.

\begin{tabular}{|c|c|c|c|c|c|c|c|c|c|c|c|c|c|}
\hline Source & & $\alpha(\mathbf{J} 2000)$ & $\delta(\mathrm{J} 2000)$ & $\begin{array}{c}F_{\mathrm{B}} \\
\mathrm{mJy}\end{array}$ & $\begin{array}{c}F_{\mathrm{R}} \\
\mathrm{mJy}\end{array}$ & $\begin{array}{c}F_{\mathrm{J}} \\
\mathrm{mJy}\end{array}$ & $\begin{array}{c}F_{\mathrm{H}} \\
\mathrm{mJy}\end{array}$ & $\begin{array}{l}F_{K_{\mathrm{s}}} \\
\mathrm{mJy}\end{array}$ & $\begin{array}{c}F_{6.75 \mu \mathrm{m}}^{a} \\
\mathrm{Jy}\end{array}$ & $\begin{array}{c}F_{15 \mu \mathrm{m}}^{a} \\
\mathrm{Jy}\end{array}$ & $\begin{array}{c}F_{24 \mu \mathrm{m}} \\
\mathrm{Jy}\end{array}$ & $\begin{array}{c}F_{70 \mu \mathrm{m}} \\
\mathrm{Jy}\end{array}$ & $\begin{array}{c}F_{850 \mu \mathrm{m}} \\
\mathrm{Jy}\end{array}$ \\
\hline $\bar{A}$ & & $23: 40: 54.65$ & $+61: 10: 27.6$ & - & - & - & - & - & - & - & 0.14 & 65 & 2.2 \\
\hline B & & $23: 40: 50.42$ & $+61: 10: 37.4$ & - & - & 0.8 & 1.5 & 1.7 & 0.2 & 0.14 & 0.16 & $<25$ & $<0.3$ \\
\hline $\mathrm{C}$ & & $23: 40: 50.25$ & $+61: 10: 27.6$ & - & - & 1.5 & 6.1 & 10.6 & $<0.02$ & 0.6 & 0.6 & $<25$ & $<0.3$ \\
\hline D & & $23: 40: 57.11$ & $+61: 10: 41.0$ & - & - & 0.07 & 0.35 & 0.7 & 0.2 & 0.1 & 0.3 & $<25$ & $<0.4$ \\
\hline E & & $23: 40: 56.35$ & $+61: 10: 30.0$ & - & - & 0.07 & 0.16 & 0.6 & 0.3 & 0.2 & 1.0 & $<35$ & $<0.8$ \\
\hline $\mathrm{F}$ & & $23: 40: 55.50$ & $+61: 10: 49.0$ & - & - & - & - & - & 0.26 & 0.2 & 0.5 & 12 & $<0.5$ \\
\hline \multirow[t]{2}{*}{ G } & & $23: 40: 52.62$ & $+61: 11: 09.2$ & - & - & 0.1 & 0.06 & 0.2 & $-^{e}$ & $-^{e}$ & 0.13 & $<30$ & $<0.5$ \\
\hline & 1 & $23: 40: 58.97$ & $+61: 10: 27.9$ & 0.1 & 0.4 & 0.07 & 0.5 & 0.5 & & & & & \\
\hline \multirow[t]{2}{*}{$\mathrm{H}^{b}$} & 2 & $23: 40: 59.04$ & $+61: 10: 33.3$ & 0.05 & 0.2 & 0.07 & 0.1 & 0.1 & $<0.01$ & $<0.15$ & 0.14 & $<20$ & $<0.2$ \\
\hline & 3 & $23: 40: 59.11$ & $+61: 10: 43.8$ & - & - & 0.8 & 0.8 & 0.6 & & & & & \\
\hline I & & $23: 40: 57.62$ & $+61: 11: 08.0$ & 0.2 & 0.7 & 6.4 & 6.6 & 5.1 & 0.24 & 0.1 & 0.08 & $-^{c}$ & $<0.2$ \\
\hline $\mathrm{L}$ & & $23: 40: 49.23$ & $+61: 11: 23.9$ & - & - & 0.64 & 2.0 & 5.5 & $-^{e}$ & $-^{e}$ & 0.3 & $-^{c}$ & $<0.1$ \\
\hline M & & $23: 40: 47.45$ & $+61: 10: 57.6$ & - & - & - & - & - & 0.04 & 0.01 & 0.33 & $<15$ & $<0.2$ \\
\hline $\mathrm{N}$ & & $23: 40: 41.44$ & $+61: 10: 28.1$ & - & - & 0.5 & 1.5 & 2.4 & $--^{e}$ & $-^{e}$ & 0.4 & $-^{c}$ & $-{ }^{d}$ \\
\hline $\mathrm{P}$ & & $23: 40: 49.23$ & $+61: 10: 11.6$ & - & - & 0.4 & 1.7 & 4.9 & 0.02 & 0.03 & 0.1 & $<15$ & $<0.4$ \\
\hline $\mathrm{R}$ & & $23: 40: 48.55$ & $+61: 10: 15.9$ & - & - & 1.0 & 1.3 & 2.1 & $-^{e}$ & $-^{e}$ & 0.08 & $<15$ & $<0.4$ \\
\hline
\end{tabular}

${ }^{a}$ From ISOCAM images (Molinari et al. 1998b).

${ }^{b}$ Although the positional association of other $K_{\mathrm{s}}$ sources to the $24 \mu \mathrm{m}$ peaks is very good (see Fig. 1b), it seems likely that source $\mathrm{H}$, since it is much shallower than the other $24 \mu \mathrm{m}$ peaks, could be associated to one of the three nearest $K_{\mathrm{s}}$ sources visible in its close proximity.

${ }^{c}$ This source is not covered by the $70 \mu \mathrm{m}$ image.

${ }^{d}$ This source is not covered by the $850 \mu \mathrm{m}$ image.

${ }^{e}$ This source is not covered by the ISOCAM images.

direct measurement and show that only a minor fraction of the total $\lambda \leq 70 \mu$ m flux density in the region can be assigned to the central core.

In addition to source $\mathrm{A}$, which is clearly dominant at $70 \mu \mathrm{m}$, we detect a fainter peak which barely emerges from the plateau in correspondence to source F; as for source A, the integrated flux is estimated using the AIPS JMFIT task fitting a peakplateau combination. Another secondary peak is visible both at 24 and $70 \mu \mathrm{m}$ about $35^{\prime \prime}$ South/South-East of A, but is considerably shallower and broader than the other sources and is not considered in this analysis. As for the other sources detected at $24 \mu \mathrm{m}$, we assigned the local values of the $70 \mu \mathrm{m}$ emission as upper limits.

The 24-70 $\mu \mathrm{m}$ spectral region alone, although very important as the SED rises toward the peak for typical YSOs envelopes, is not sufficient for a reliable comparison with SED models. Leaving aside IRAS whose beam at $100 \mu \mathrm{m}$ encompasses more than the entire area shown in Fig. 1a, the longer wavelength information is extracted from submillimeter and millimeter images (Molinari et al. 2002). Similarly to the MIPS $70 \mu \mathrm{m}$ image, the $850 \mu \mathrm{m}$ SCUBA image shows an extended emission patch covering the entire area, in which a strong peak coincident with source A clearly stands out; $850 \mu$ m fluxes for the various $24 \mu \mathrm{m}$ sources were assigned following the same approach as for the $70 \mu \mathrm{m}$ fluxes. The peak at source A is also exactly coincident with the core visible in the interferometric OVRO images at $3.4 \mathrm{~mm}$; the slight offset of the $70 \mu \mathrm{m}$ peak position with respect to the $24 \mu \mathrm{m}$ source A-peak may then not be real and could be ascribed to an inaccuracy of the $70 \mu \mathrm{m}$ image astrometry. 


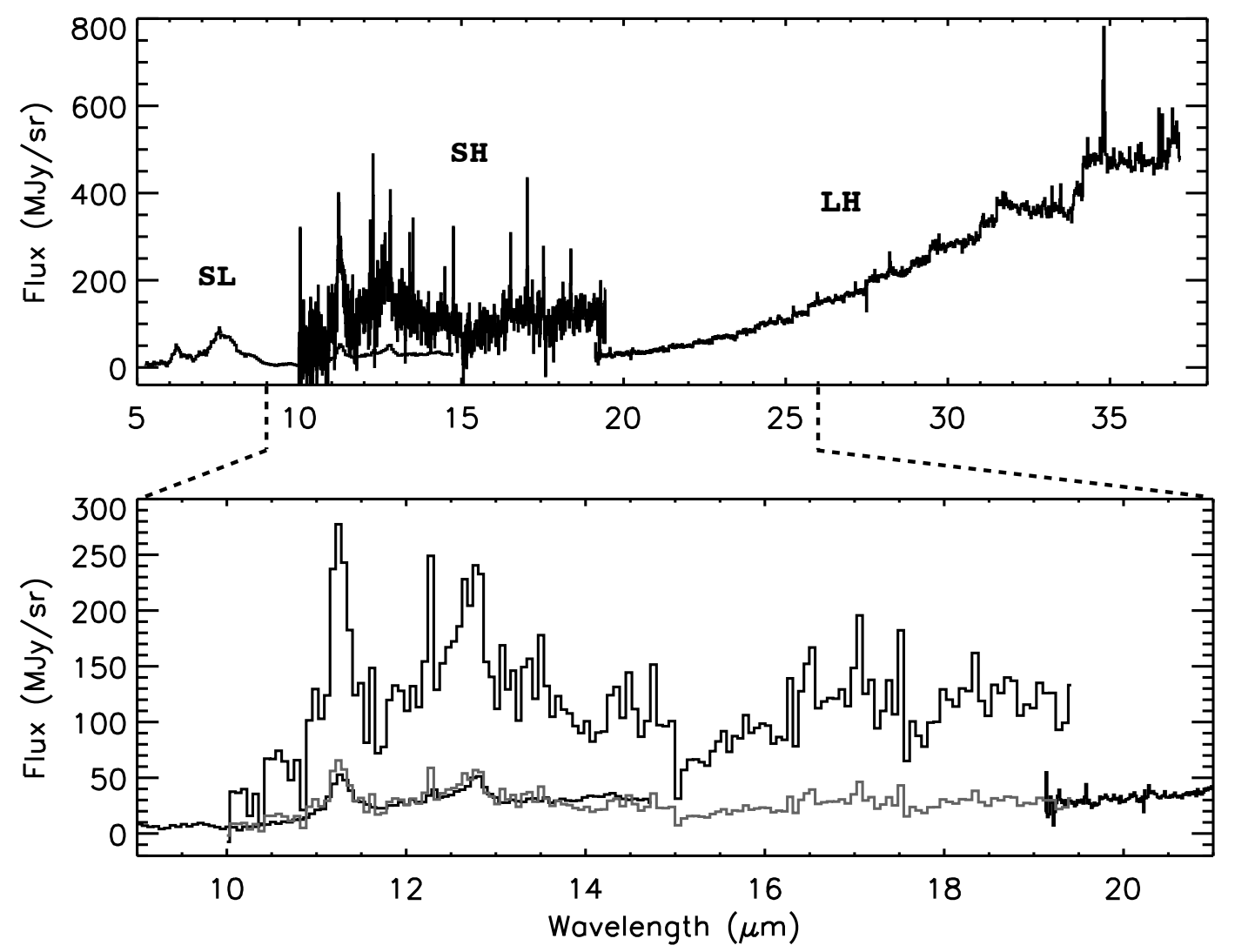

Fig. 2. Full IRS spectrum (top panel) extracted using CUBISM at the position of the central millimeter core (source A). The bottom panel is an enlargement where this time the SH module spectrum is resampled at the resolution of the SL module; its rescaling by a factor of 4.2 brings it to an almost perfect overlap with the SL spectrum, and joins very well with the LH spectrum at longer wavelength.

Concerning the shorter wavelengths, for most of the sources we could estimate flux densities at 6.75 and $15 \mu \mathrm{m}$ from ISOCAM images (Molinari et al. 1998b) using the same approach followed for the MIPS $24 \mu \mathrm{m}$ image; sources $\mathrm{G}, \mathrm{L}, \mathrm{N}$ and $\mathrm{R}$ are outside the ISOCAM FOV. Near-IR counterparts in $J, H$, and $K_{\mathrm{s}}$ could also be assigned for most of the sources (Faustini et al., A\&A, submitted). The $K_{\mathrm{s}}$-image is shown in greyscale in the right panel of Fig. 1; the full-line contours report the $24 \mu \mathrm{m}$ emission, while the dashed contours mark the emission pattern in the $3.6 \mathrm{~cm}$ thermal free-free emission (Molinari et al. 2002). The only dubious counterpart assignment concerns source $\mathrm{H}$, for which there are three NIR objects very close to the $24 \mu \mathrm{m}$ peak; we list all of them in Table 1 .

For those $24 \mu \mathrm{m}$ sources with NIR counterpart we tried to extend the SED coverage to the visible range by visual inspection of the Digital Sky Survey 2 blue and red plates. Where counterparts could be found, fluxes were estimated doing standard aperture photometry and calibrating the integrated flux using a conversion factor estimated from the magnitude of medium intensity sources in the field as reported in the USNO-B1 cata$\log$. Zero-magnitude fluxes of $4260 \mathrm{Jy}$ and $3080 \mathrm{Jy}$ for the $B$ and $R$ bands were used (Bessel 1979).

\subsection{IRS spectroscopy}

The areas observed in the $3 \times 2$ spectroscopic map are different across the wavelength range since the slits in the different modules have different sizes and orientations; however they are much smaller than the area imaged by MIPS. All slits were centered on the millimeter peak position (coincident with source A), so that we have complete spectral coverage for this source. We used the CUBISM software (Smith et al. 2007) to mosaic and co-add the spectra from all the observed positions and obtain convenient spectral cubes for further analysis. We present in Fig. 2 the complete spectrum in surface brightness units extracted at the position of source A (top panel).

Spectra from the two SL1 and SL2 modules for $\lambda \leq$ $15 \mu \mathrm{m}$ have been merged together and clearly show a set of Poly-Aromatic Hydrocarbon (PAH) features at wavelengths between 6 and $13 \mu \mathrm{m}$. The SH spectrum is particularly noisy not only with respect to the lower resolution SL portion, but also with respect to the LH. Besides, it is also apparent (see bottom panel of Fig. 2) that the SL and LH portions appear to be quite well aligned among them, while the SH portion seems anomalous in this respect. To understand the nature of the displacement of SH we resampled it to the same resolution as the SL portion, with which SH overlaps between 10 and $15 \mu \mathrm{m}$; the resampled $\mathrm{SH}$ is shown in black in the bottom panel of Fig. 2; normalising it to the SL over the entire overlapping range we obtain a ratio of 4.2 , and if we rescale $\mathrm{SH}$ by this factor (the grey line in the figure) we see that SH almost perfectly overlaps with SL. We conclude that the SH spectrum likely suffers from an incorrect calibration, and a "gain" type of correction brings it in excellent agreement with both SL and LH. We then rescale SH by this gain factor, and we will use this rescaled spectrum to extract lines and estimate integrated fluxes.

Figure 3 shows six lines identified in the SH and LH spectra. [NeII]12.8 $\mu \mathrm{m}$ line and the $\mathrm{S}(1)$ and $\mathrm{S}(2)$ pure rotational 


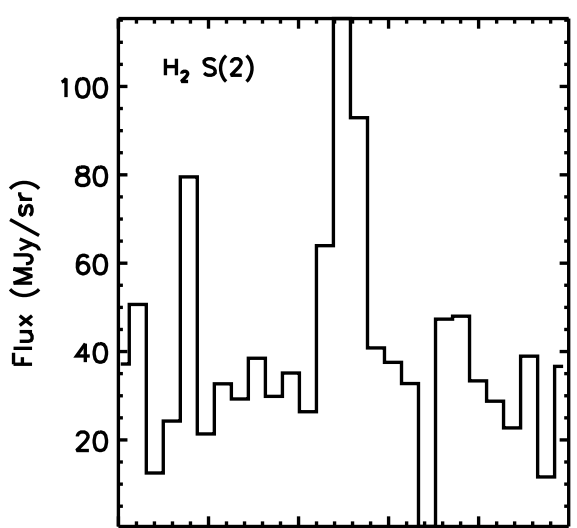

$12.1512 .2012 .25 \quad 12.3012 .35$

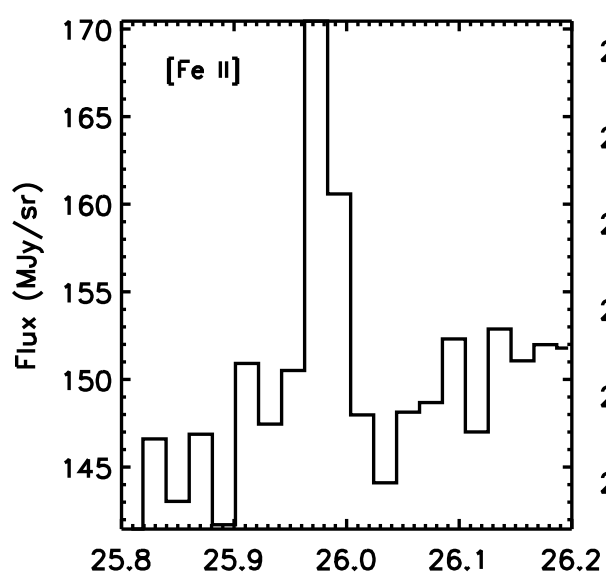

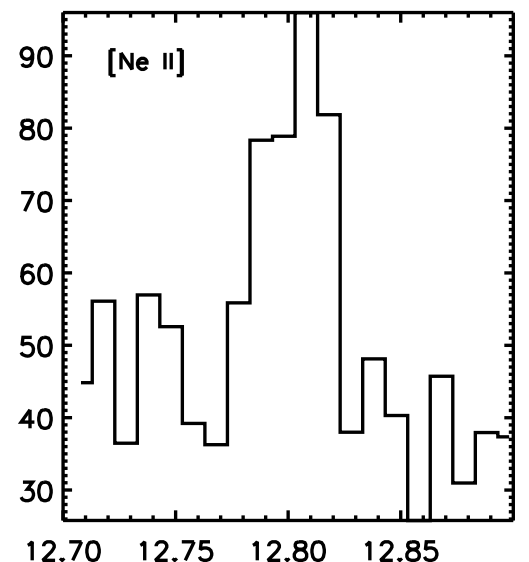

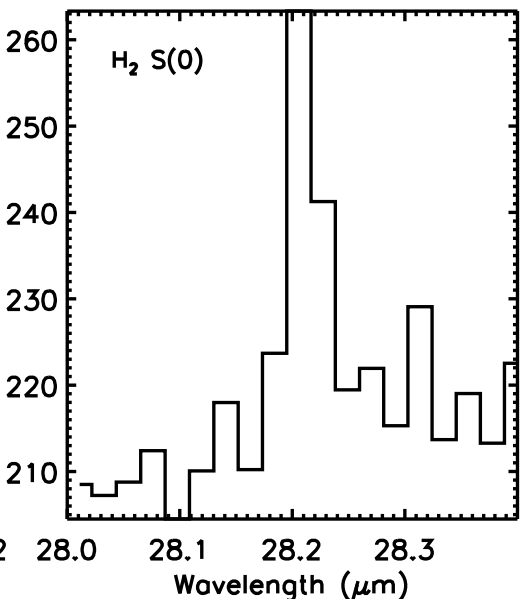

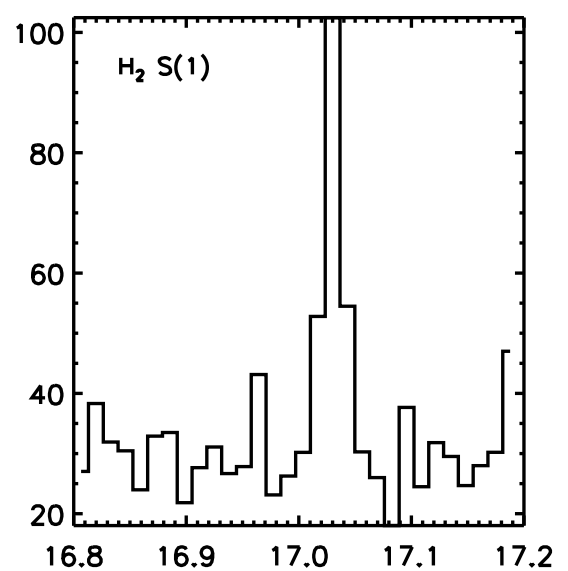

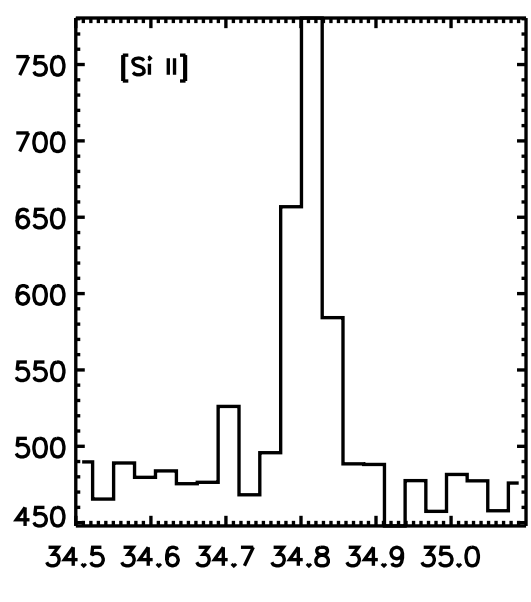

Fig. 3. The six identified lines in the entire spectrum at the position of source A, as extracted from the spectrum in Fig. 2; the various lines are identified in each panel.

Table 2. Integrated line fluxes at the position of source A.

\begin{tabular}{lcc}
\hline \hline Line & $\begin{array}{c}\text { Wavelength } \\
(\mu \mathrm{m})\end{array}$ & $\begin{array}{c}\text { Flux } \\
\mathrm{W} \mathrm{cm}^{-2} \mathrm{sr}^{-1}\end{array}$ \\
\hline $\mathrm{H}_{2}(0-0) \mathrm{S}(0)$ & 28.2 & $6.4 \times 10^{-13}$ \\
$\mathrm{H}_{2}(0-0) \mathrm{S}(1)$ & 17.02 & $1.7 \times 10^{-12}$ \\
$\mathrm{H}_{2}(0-0) \mathrm{S}(2)$ & 12.28 & $3.2 \times 10^{-12}$ \\
{$[\mathrm{NeII}]$} & 12.8 & $3.2 \times 10^{-12}$ \\
{$[$ FeII $]$} & 26 & $3.5 \times 10^{-13}$ \\
{$[\mathrm{SiII}]$} & 34.8 & $4.3 \times 10^{-12}$ \\
\hline
\end{tabular}

lines of $\mathrm{H}_{2}$ are extracted from the $\mathrm{SH}$ spectrum; the LH spectrum is relatively less noisy and allows us to reliably identify the [FeII] $26 \mu \mathrm{m}$, the $\mathrm{H}_{2} \mathrm{~S}(0)$ and the [SiII] $34.8 \mu \mathrm{m}$ lines. Integrated fluxes were derived using Gaussian fits to line profiles after subtraction of linear baselines; estimated values are reported in Table 2 for the position of source A.

\section{Source A: an accreting pre-ZAMS massive YSO}

Source A is the most noticeable object in this region. While undetected in the near-IR and ISOCAM images, it is first visible at $24 \mu \mathrm{m}$ and then becomes the most prominent source in the far-IR, the submillimeter and the millimeter regions, and it coincides with the location of the driving source of the molecular outflow seen in $\mathrm{HCO}^{+}(1 \rightarrow 0)$ and $\mathrm{SiO}(v=0,2 \rightarrow 1)$ (Molinari et al. 1998b).
Source A, however, is not as prominent in the far-IR (Fig. 4) as we originally believed when only IRAS data were available (Fontani et al. 2004). This has very important consequences regarding its nature.

In a systematic analysis of the SEDs of a sample of 42 sites of massive star formation (Molinari et al. 2008) we used the radiative transfer models of Whitney et al. (2003) to compute a grid of SEDs for embedded ZAMS stars of various spectral classes (from B5 to O3) and for a wide range of envelope properties (radius, mass, geometry). In that work the far-IR portion of the SED was based on IRAS fluxes (the diamonds in Fig. 4), corrected for a possible contribution from extended emission. We could not provide a successful fit with any of the models of our grid for what we now call source A in Mol160/IRAS 23385+6053. Likewise, using the much more reliable estimate of the source SED in the far-IR range that the MIPS data allow (the squares in Fig. 4), we still cannot find any acceptable fit with models of embedded ZAMS stars; any model that fits the $850 \mu \mathrm{m}$ and $3.4 \mathrm{~mm}$ points predicts far too much flux shortward of $100 \mu \mathrm{m}$. We neglected the possible contribution of free-free emission to the $3.4 \mathrm{~mm}$ flux as this could amount to a 10-20\% level at most, given that source A was not detected at $2 \mathrm{~cm}$ at the VLA in the B-configuration (Molinari et al. 1998a), and in the extreme case of optically thick free-free $\left(F \propto v^{2}\right)$ up to the millimeter range.

The full line in Fig. 4 represents the best fit, using a simple greybody which assumes constant density and temperature. The fit uses the OVRO, SCUBA, and MIPS data, and the ISOCAM 


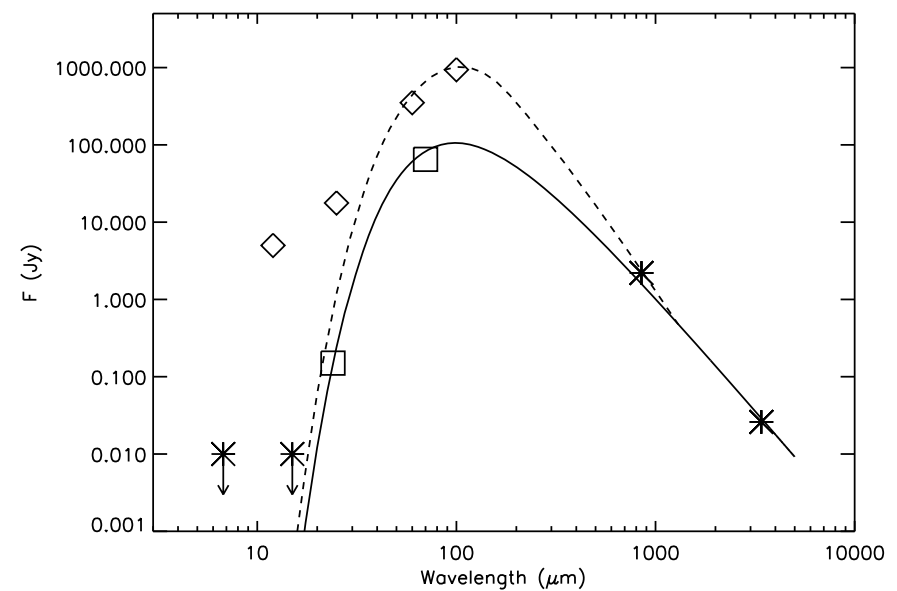

Fig. 4. Spectral Energy Distribution (SED) for the central core of Mol160/IRAS 23385+6053. The diamonds are the IRAS fluxes, the squares are the MIPS fluxes and the asterisks represent the ensemble of measurements from ISOCAM (upper limits since there is no detection below $24 \mu \mathrm{m}$ ), SCUBA and OVRO; the dashed line is a greybody fit to the above data set using the IRAS 60 and $100 \mu$ m fluxes (as in Molinari et al. 1998b). The full line is a greybody fit to the SED using the MIPS data instead of the IRAS ones.

upper limits (the asterisks) but neglects the 12 and $25 \mu \mathrm{m}$ IRAS data since they are clearly due to the other YSOs in the region (see Fig. 1). The overall fit is remarkably good and corresponds to an envelope with $T=37 \mathrm{~K}, M=220 M_{\odot}$ and a dust opacity index $\beta=1$, assuming a dust opacity of $0.005 \mathrm{~cm}^{2} \mathrm{~g}^{-1}$ at $1.2 \mathrm{~mm}$ and a gas-to-dust ratio of 100 (Preibisch et al. 1993).

Robitaille et al. (2006) used the same radiative transfer code of Whitney et al. (2003) that we use in Molinari et al. (2008), to produce a much more extensive grid of SED models (not yet available at the time of our previous work) including central stars in all pre-MS phases from the birthline to the ZAMS. It is quite convenient to use the automatic SED fitting tool provided by Robitaille et al. (2007), which reports the best fitting models in increasing $\chi^{2}$ order. Fitting the SED of source A with this grid of models provides a best fit for a central object of $\sim 9.5 M_{\odot}$ with a radius of $\sim 25 R_{\odot}$ and a surface temperature slightly in excess of $8000 \mathrm{~K}$ accreting at $\sim 2 \times 10^{-3} M_{\odot} \mathrm{yr}^{-1}$ from a massive $\sim 500 M_{\odot}$ envelope. This value of the stellar radius is much higher than typical values for ZAMS stars of that mass; besides, with these accretion rates the stellar mass needed to ignite deuterium burning is higher than $20 M_{\odot}$ (Palla \& Stahler 1992), so that such an object would still be in the pre-ZAMS phase. Clearly, given the number of parameters involved in the modelling and the limited number of data points available, the fitting cannot pinpoint a unique combination of parameters but rather identifies a certain range in the parameter space. It is true, however, that the possibility of an embedded ZAMS star seems to be reliably excluded and that the above-mentioned combination of parameters is representative of the region of the parameter space identified by the SED fitting.

The greybody fit represented by the full line in Fig. 4 provides an integrated bolometric luminosity of $3170 L_{\odot}$ at a distance of $4.9 \mathrm{kpc}$ (similar to what was obtained by Stecklum et al. 2005). This is remarkably lower than the $\sim 16000 L_{\odot}$ obtained again fitting a greybody but using the IRAS PSC 60 and $100 \mu \mathrm{m}$ fluxes (the dashed line in Fig. 4, see also Molinari et al. 1998b), or than the $\sim 10000 L_{\odot}$ obtained if we try to correct the far-IR fluxes for extended emission using higher spatial resolution information from the submillimeter, as done in

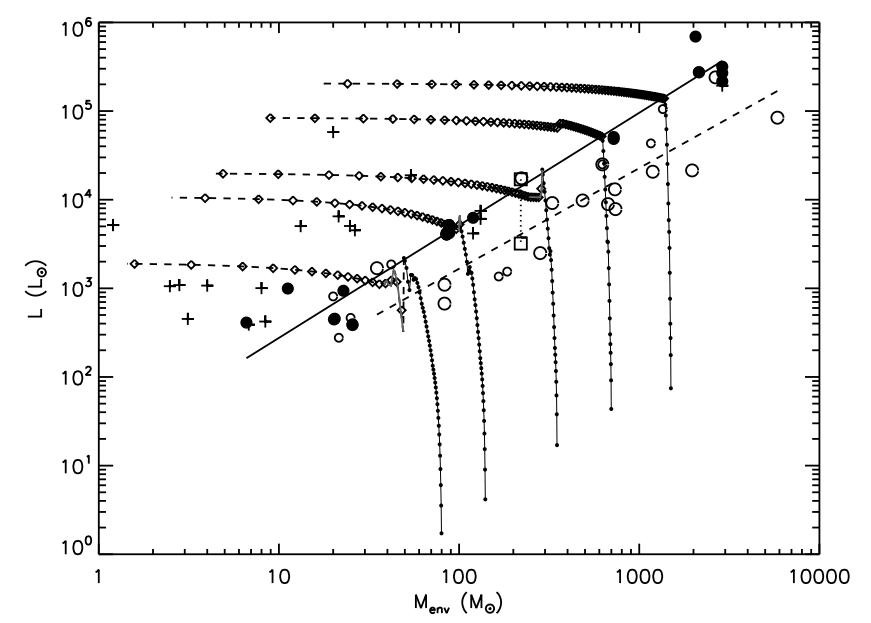

Fig. 5. $L_{\mathrm{bol}}-M_{\mathrm{env}}$ diagram from Molinari et al. (2008); full circles are IR-P sources, empty circles are MM-P sources and plusses are for IR-S sources. Dotted full lines and diamond dashed lines are evolutionary models; the two squares connected by the vertical dotted line represent the extent of the shift of source A due to the new luminosity estimate. The full and dashed diagonal lines represent a linear fit to the position of the IR-P and MM-P sources, respectively.

Molinari et al. (2008). Thanks to the MIPS data we can now set much more stringent constraints on the SED over the entire wavelength range and we can obtain this much lower value for the luminosity while the estimated envelope mass remains basically the same.

This decrease in the luminosity estimate has important consequences concerning the evolutionary stage of the massive forming object. In our recent analysis of the SED evolution of massive YSOs (Molinari et al. 2008) we could differentiate two classes of objects depending on whether the entire SED from the mid-IR to the millimeter can be consistently fitted with a single model of an embedded ZAMS star (called "IR-P" by Molinari et al. and represented with the filled circles in Fig. 5) or not. If not, the SED was modeled with two components: a singletemperature greybody for the millimetric/far-IR part ("MM-P", open circles in Fig. 5), and a mildly-obscured ZAMS star for the mid-IR ("IR-S", plus signs in Fig. 5). These different classes of objects occupy distinct regions in the $L_{\mathrm{bol}}-M_{\mathrm{env}}$ diagram (Fig. 5), and a simple toy-model for the evolution of the bolometric luminosity and the circumstellar envelope can explain the sequence MM-P/IR-P/IR-S in evolutive terms analogous to the Class 0-I-II sequence established for low-mass YSOs. From a statistical viewpoint the IR-P objects are dominated by sources with far-IR colors of Hot Cores/UCHII regions, therefore confirming their ZAMS nature; this is not the case for MM-P objects. In addition to the SED differences, Fig. 5 shows that the luminosity is a critical parameter that can trace with a high dynamical range the transition between MM-P and IR-P objects.

The two squares in Fig. 5 illustrate the position of the source with the luminosity that was found in our original work on this source (Molinari et al. 1998b) and the one determined using the MIPS observations. Quite remarkably, the shift to lower luminosity brings the source from the locus of the IR-P objects (the full straight line, which in our interpretation marks the arrival onto the ZAMS) to the locus of the MM-P objects (the dashed straight line). In evolutionary terms within the framework of the model proposed in Molinari et al. (2008), this luminosity correction makes the source more than $5 \times 10^{4}$ years younger than we have previously estimated; this is the time it takes, according 


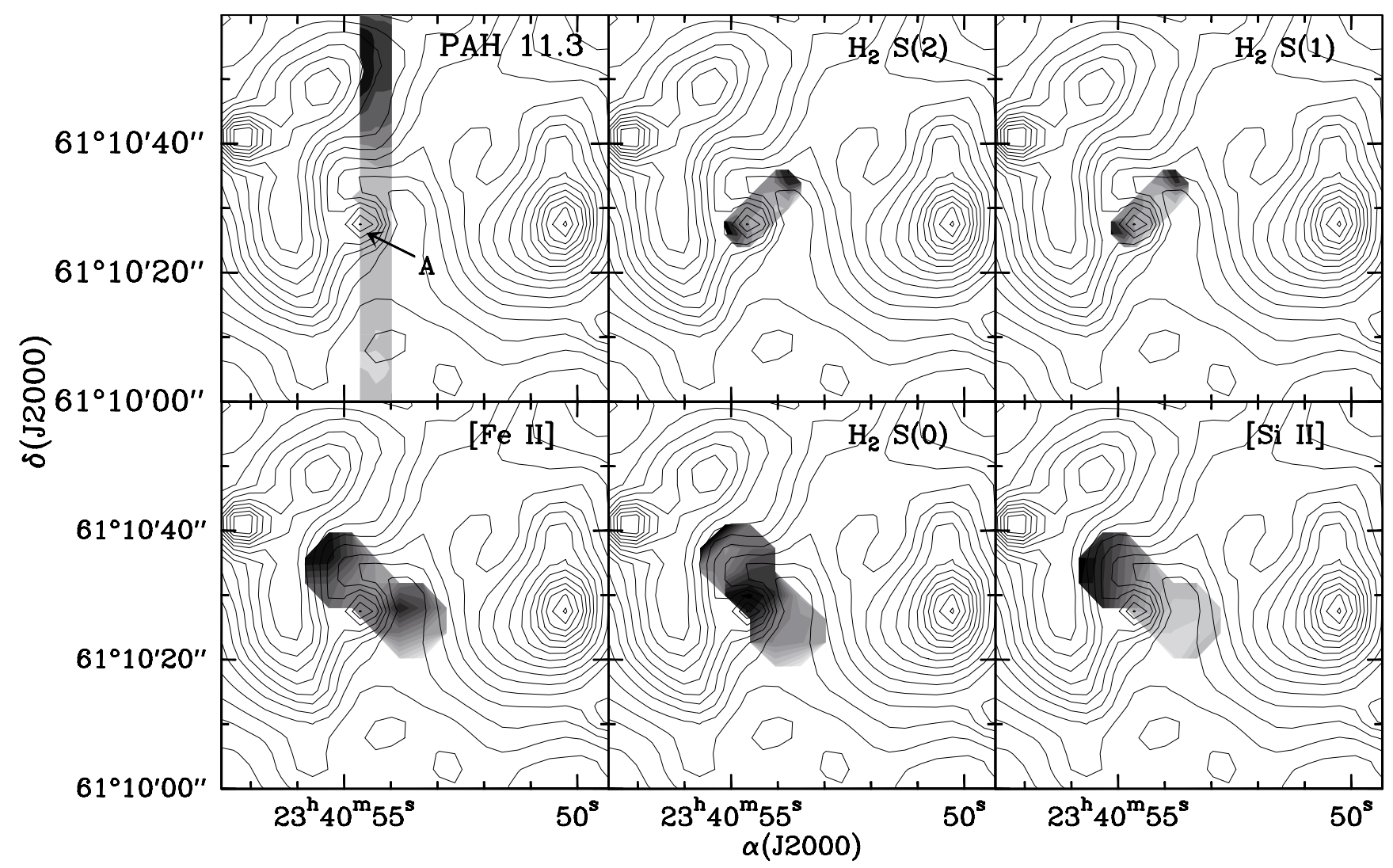

Fig. 6. Grey-scale maps (intensity increases from white to black) of continuum-subtracted integrated line intensities superimposed on the contours of the $24 \mu \mathrm{m}$ continuum emission. The various panels show, from left to right and from top to bottom: the PAH $11.3 \mu \mathrm{m}$ feature, $\mathrm{H}_{2} \mathrm{~S}(2), \mathrm{H}_{2} \mathrm{~S}(1)$, [FeII] $26 \mu \mathrm{m}, \mathrm{H}_{2} \mathrm{~S}(0)$, and [SiII] $34.8 \mu \mathrm{m}$. The map for the [NeII] $12.8 \mu \mathrm{m}$ line is not reported but the emission distribution is virtually identical to the $\mathrm{H}_{2} \mathrm{~S}(1)$ and $\mathrm{S}(2)$ lines.

to the prescriptions of accelerating accretion models (McKee \& Tan 2003) to bring source A onto the ZAMS from its present location in the $L_{\mathrm{bol}}-M_{\mathrm{env}}$ diagram. Source $\mathrm{A}$ is even younger if we adopt the $\sim 500 M_{\odot}$ envelope mass derived from the detailed grid of SED models of Robitaille et al. (2006).

Molinari et al. (2008) also suggested that MM-P objects are characterized by a much steeper SED for $\lambda \leq 100 \mu \mathrm{m}$ compared to IR-P objects. In particular we predicted that the [24-70] color (estimated by interpolating observed mid-IR and sub-mm spectra using SED modeling) to be $\sim 1$ for IR-P objects and $\sim 4$ for MM-P objects: the MIPS observations provide a direct measurement of the SED shape in this critical region: with [24-70] $=2.6$ for source $A$, these measurements strenghten the foundations for the working assumption in our previous work.

\section{Other YSOs and the UV-field in the region}

Since source A is only detected longward of $24 \mu \mathrm{m}$ in the continuum, dust extinction will prevent any line emission at shorter wavelengths, originating from the embedded YSO, to emerge and be detectable. Therefore all PAH features, the [NeII] $12.8 \mu \mathrm{m}$, and the two $\mathrm{S}(2)$ and $\mathrm{S}(1)$ lines cannot originate from the massive central core; indeed all emission features are detected with variable intensities throughout the regions mapped with the IRS. To better investigate their spatial distribution we again used CUBISM to produce continuum-subtracted spatial maps of the integrated line fluxes. They are presented in Fig. 6 for the $11.3 \mu \mathrm{m}$ PAH feature and for five of the detected lines, as indicated.
The intensity of the $11.3 \mu \mathrm{m}$ feature (the same spatial distribution is followed by all other PAHs) has a minimum at the location of source A and increases towards the north where source $\mathrm{F}$ is located. PAHs emit in a non-equilibrium regime in the presence of relatively energetic incident radiation field. Their detection, with integrated intensities similar to that of the underlying continuum, suggests then the presence of an irradiating UV-field in the region. Indeed, all detected transitions are commonly found in regions of intense UV-irradiation where the local ISM is photo-dissociated ([FeII $] 26 \mu \mathrm{m}$, [SiII $] 34.8 \mu \mathrm{m}$ plus the three $\mathrm{H}_{2}$ lines; see Tielens \& Hollenbach 1985; and Kaufman et al. 1999) or photo-ionized ([NeII]12.8 $\mu \mathrm{m}$, see Morisset et al. 2002), consistent with the spatial distribution of the continuum-subtracted integrated emission for all detected lines. In all panels of Fig. 6 the grey-scale shows, with the exception of $\mathrm{H}_{2} \mathrm{~S}(0)$, that the intensity of the continuum-subtracted integrated line fluxes increases away from source A. We will discuss this in more detail below, but we anticipate that this distribution seems to rule out any significant role of source $A$ in the budget of the UV-field in this region. The $\mathrm{H}_{2}$ rotational lines are more commonly interpreted as being due to C-shocks, but the extended distribution observed seems more consistent with a PDR origin. The line ratio analysis using all the detected lines in Fig. 3 was carried out using the PDR Toolbox ${ }^{1}$ that conveniently implements a web-based fitting program using the diagnostic diagrams based on the models by Kaufman et al. (1999). Metallicities 2-3 times higher than local ISM values, and more typical of

\footnotetext{
1 The PDR Toolbox is at http://dustem.astro.umd.edu/pdrt/ index.html
} 
HII regions and massive star forming regions in general (Shaver et al. 1983; Tielens \& Hollenbach 1985) have been used. Most of the line ratios are consistent with a far-UV field intensity of the order of $100 \leq G_{0} \leq 1000$, in units of the FUV field intensity in the solar neighborhood (Habing 1968), for an ISM density in excess of $10^{4} \mathrm{~cm}^{-3}$. The lines which deviate most from defining a consistent picture are the $\mathrm{H}_{2} \mathrm{~S}(2)$ and the [FeII] $26 \mu \mathrm{m}$, which appear to be a factor of 2-3 stronger than PDR models predictions given the fluxes of the other lines. The modeled ISM densities are entirely reasonable for the intra-clump material of a massive star forming region, as shown from maps in high-density tracing species like $\mathrm{HCO}^{+}(1-0),{ }^{13} \mathrm{CO}(2-1)$ and $\mathrm{CS}(3-2)$ (Brand et al. 2001).

To verify if external field objects could possibly be responsible for the detected UV-irradiation, we searched the Spectral Classification catalogs available in Aladin for stars with spectral type earlier than B. The nearest object is the B9V star BD+60 2600 located more than 10' to the North; assuming standard stellar parameters (Thompson 1984) and a distance equal to Mol160/IRAS $23385+6053$ the resulting $G_{0}$ is few orders of magnitudes below the observed levels.

Among the various detected sources in this region, source A is the most massive and luminous; its extreme optical depth below $20 \mu \mathrm{m}$, however, would in principle allow only [FeII] $26 \mu \mathrm{m}$, [SiII] $34.8 \mu \mathrm{m}$ and $\mathrm{H}_{2} \mathrm{~S}(0)$ to escape from its inner envelope regions. However, the only indication we have in this respect, as already noted, is limited to the $\mathrm{H}_{2} \mathrm{~S}(0)$ which however traces warm gas in general; the former two lines, that are the most commonly used PDR tracers, are instead peaking away from source A. The fact that also the intensity of the PAH feature, which is directly related to the intensity of the UV-field, increases away from source A confirms that the latter cannot be the radiating source of the UV-field, not even in the presence of a clumpy circumstellar envelope.

The other sources detected in the region are much brighter than A at $24 \mu \mathrm{m}$ and many have a near-IR counterpart, so that they could be intermediate mass YSOs in a more evolved state than source A. However, given the limited spatial coverage of the IRS maps, it is not possible to use spectroscopic tools to get more insight into the nature of these various objects.

More can be learned from the SED modelling of the other sources revealed at $24 \mu \mathrm{m}$ (Table 1). Now, we seek evidence for the presence of an embedded ZAMS star, as opposed to a pre-MS object, which might be responsible for the relatively intense UV-irradiation conditions in the region, as well as for the extended radio continuum which is thermal free-free in origin (see Fig. 1b). We again used the automatic SED fitting tool of Robitaille et al. (2007). For sources with no counterpart in the near-IR or in the visible, we adopted the limiting magnitudes of our own $J H K_{\mathrm{s}}$ data (Faustini et al., submitted) and of the DSS2 plates. We searched, among the various $\chi^{2}$-ranked fits, for models where the central stars have photospheric temperatures and stellar radii typical of ZAMS stars, to verify if some of the sources detected in the region could be capable of radiating sufficient quantities of UV flux to photo-dissociate the intraclump medium as suggested by the IRS spectroscopy. Such models could be found for sources B, C, D, H and I, although not with the best formal $\chi^{2}$; they rank lower in the best $\chi^{2}$ list, but still provide very good fits (shown in Fig. 7). No such fits could instead be found for the other sources in Table 1, which can only be fitted with pre-ZAMS objects. Table 3 reports the model parameters for SED of embedded ZAMS stars; Col. 9 lists the Lyman continuum as tabulated by Thompson (1984) for ZAMS stars with photospheric temperatures similar to those reported in Col. 3. Column 8 reports the model intrinsic bolometric luminosity. The sum of the values in Col. 8 plus the luminosity previously estimated for source A amounts to $\sim 30000 L_{\odot}$ which is $50 \%$ higher than the value obtained for the entire region using the IRAS fluxes; we regard this discrepancy as barely significant, given the spread of the model luminosity depending on the exact model selected in the fit. Column 10 shows the unattenuated integrated $6-13.6 \mathrm{eV}$ far-UV continuum, expressed in units of the far-UV field intensity in the solar neighborhood (Habing 1968), irradiated by each source at the position of source A.

The total $G_{0}$ at the position of source A is consistent with the regime deduced from the infrared IRS lines, if PDR in origin. Since $G_{0}$ in Col. 10 of Table 3 is computed with no intervening attenuation between the emitting source and the position of source A, we must verify that this is indeed plausible. Indeed, all the models in Table 3 are obtained for systems where the ZAMS star is surrounded by a disk with high inclination angle with respect to the line-of-sight, and with no envelope. The SEDs which fit the observed data points (see Fig. 7) are obtained assuming an additional extinction correction along the line-of-sight to the observer (Col. 7) due to intervening dust not related to the immediate circumstellar environments. Since these values are not homogeneous, and the distribution of optically visible objects in the Mol160/IRAS 23385+6053 area is not suggestive of significant or variable interstellar absorption, we believe this extinction to be intra-cluster in origin. It is clear that with such amounts of extinction the far-UV field should be entirely absorbed, so that we have to assume that the line-of-sight from the various sources to source A must be relatively dust-free. That this must be the case also seems to be suggested by the distribution of the radio continuum from thermal free-free emission (dashed lines in Fig. 1b); the extent of the emission requires that sources of ionizing continuum must be present, and that this continuum is not confined to the immediate surroundings of the ionizing stars. The most likely sources for the radio emission seem to be sources D and $\mathrm{H}$ for the eastern radio lobe, and sources $\mathrm{B}$ and $\mathrm{C}$ for the western radio lobe.

Concerning the intensity of the radio emission, however, its conversion into unattenuated Lyman continuum intensity $N_{\mathrm{Ly}}$ provides values (Molinari et al. 2002) one order of magnitude higher than those that can be justified by the modeled ZAMS objects in the region (Col. 9 of Table 3). Even integrating the IRAS and millimeter fluxes would yield a value of $\sim 20000 L_{\odot}$ which, also if coming from a single ZAMS star (which is not the present case), is again not sufficient to provide the amount of $N_{\text {Ly }}$ deduced from the radio fluxes. It should also be noted that the latter is a lower limit because the free-free emission has been assumed optically thin and possible attenuation from dust inside the HII regions has not been considered. The simplest way to reconcile this apparent inconsistency would be to posit that the distance of $4.9 \mathrm{kpc}$ which we assumed for this object is incorrect. Indeed, while the bolometric luminosity scales with the square of the distance, the relationship between ZAMS luminosity and Lyman continuum is much steeper. In the limiting case of a single object emitting all the luminosity (which is unrealistic given the evidence), moving the object to a distance of $\sim 6 \mathrm{kpc}$ would raise the luminosity to $\sim 29000 L_{\odot}$ which would produce $\log \left(N_{\text {Ly }}\right) \sim 47.4$; this is equal to the $N_{\text {Ly }}$ deduced from the observed radio flux rescaled to a distance of $6 \mathrm{kpc}$. The evidence from the radio continuum, however, is that there are at least two major sources of the ionizing-flux field; a simple calculation shows that in the limit of two dominant ZAMS objects the distance should be increased to about $8 \mathrm{kpc}$. The $V_{\mathrm{LSR}}$ that 

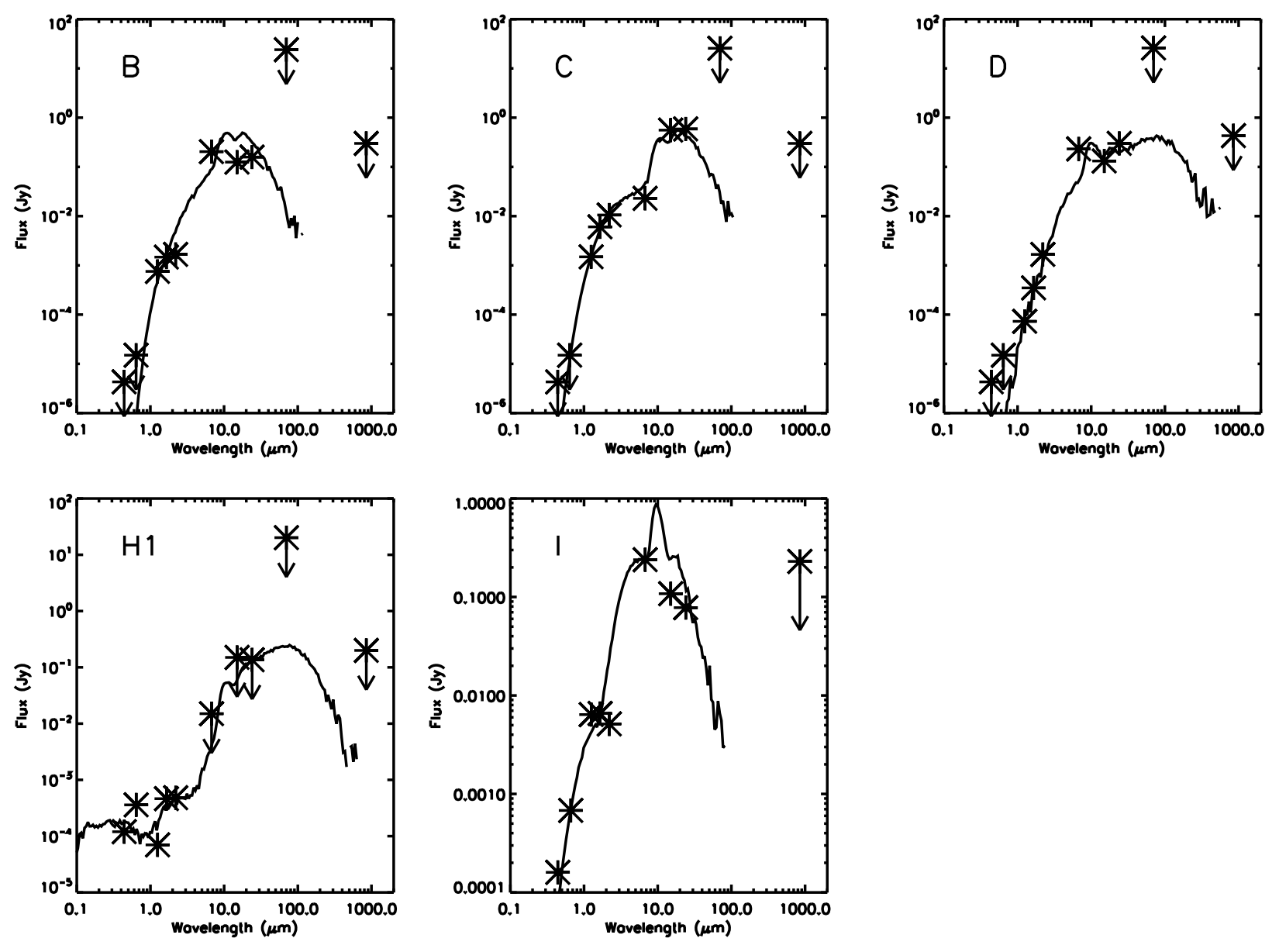

Fig. 7. SEDs for the various sources where a fit with a model of an embedded ZAMS star could be found.

Table 3. Models results for candidate ZAMS sources in the field.

\begin{tabular}{lccccccccc}
\hline \hline Sou. & $\begin{array}{c}M_{\star} \\
{\left[M_{\odot}\right]}\end{array}$ & $\begin{array}{c}T_{\star} \\
{[\mathrm{K}]}\end{array}$ & $\begin{array}{c}R_{\star} \\
{\left[R_{\odot}\right]}\end{array}$ & $\begin{array}{c}R_{\text {Disk }} \\
{[\mathrm{AU}]}\end{array}$ & $\begin{array}{c}i \\
{\left[{ }^{\circ}\right]}\end{array}$ & $\begin{array}{c}A_{\mathrm{V}} \\
{[\mathrm{mag}]}\end{array}$ & $\begin{array}{c}L_{\text {bol }}^{a} \\
L_{\odot}\end{array}$ & $\begin{array}{c}\log \left[N_{\mathrm{Ly}}\right] \\
{\left[\mathrm{s}^{-1}\right]}\end{array}$ & $G_{0}$ \\
\hline $\mathrm{B}$ & 11.4 & 27500 & 4.3 & 380 & 87 & 15.5 & 9400 & 46.3 & 200 \\
$\mathrm{C}$ & 9.7 & 25300 & 3.9 & 2000 & 70 & 11.5 & 5600 & 45.8 & 120 \\
$\mathrm{D}$ & 10.0 & 25600 & 4.0 & 660 & 87 & 7.3 & 6400 & 45.9 & 135 \\
$\mathrm{H}$ & 6.0 & 18400 & 2.9 & 410 & 87 & 0.0 & 900 & 43.4 & 10 \\
$\mathrm{I}$ & 9.2 & 24500 & 3.8 & 120 & 70 & 6.2 & 4700 & 45.8 & 50 \\
\hline
\end{tabular}

${ }^{a}$ As given by the models.

would be implied by this distance is more than $20 \mathrm{~km} \mathrm{~s}^{-1}$ different from the value measured from radio spectroscopy, and this is not incompatible with the magnitude of the streaming motions deduced from the observed velocity field (Brand \& Blitz 1993).

Changing the distance of this region to $8 \mathrm{kpc}$ does not affect our interpretation about the nature of source $A$ as it emerges from Fig. 5; both envelope mass and luminosity scale the same way with distance so that the position of source A in Fig. 5 would shift toward upper-right along a line of slope 1, therefore leaving the source in the area occupied by MM-P objects. This higher distance would also imply that the mass regime of the Mol160/IRAS 23385+6053 region is higher than previously thought; in particular source A, which in our proposed evolutionary interpretation and at the distance of $4.9 \mathrm{kpc}$ would reach the ZAMS (the solid line corresponding to the location of the IR-P objects in Fig. 5) at a luminosity of nearly $16000 L_{\odot}$ as a B0.5 star, if placed at the distance of $8 \mathrm{kpc}$ would reach the ZAMS at about $40000 L_{\odot}$, roughly corresponding to an O9.5 star.

\section{Conclusions}

Sub-arcminute spatial resolution in the far-IR proves to be critical for a proper assessment of the evolutionary stage of a massive forming object. SED modeling of the most prominent YSOs in the Mol160/IRAS 23385+6053 region suggests the presence of several intermediate and high-mass YSOs in different evolutionary stages. While some of them are compatible with being ZAMS objects with spectral types between B1.5 and B5, source A is best interpreted as a strongly accreting object of central mass comparable to the other ZAMS YSOs in the area, but not yet on the ZAMS. The region immediately surrounding source A is bright in infrared lines which are typical of photodissociation/ionisation regions; the deduced intensity of the radiative far-UV field at the position of source A is compatible with emission from the few ZAMS objects as characterized by the SED modelling, which are also likely to be responsible for the extended radio continuum emission. There is an inconsistency, however, between the Lyman continuum estimated from the radio flux and from the luminosity of the modeled ZAMS stars in the region. At the moment we can only reconcile this if we assume that the region is almost twice as distant as assumed up to now, which obviously would need independent confirmation.

The extreme values of the circumstellar extinction implied by the extreme SED of source A exclude that this source can participate in any way to the far-UV radiative field which permeates the region. As a whole, the population of intermediate 
and massive YSOs in the region is suggestive of a star formation timescale of the order of few $10^{5}$ years, as suggested by models.

However, intermediate- and high-mass stars are not the only objects found in this region. A cluster of lower-mass stars is revealed in the near-IR; this is partially apparent in Fig. 1b, where the contrast, however, is not optimal to visually reveal the much more abundant population of fainter objects. Stellar density analysis shows the cluster to be as extended as the extent of the mid- and far-IR emission, with several tens of members (Faustini et al., submitted). The comparison of the $K$-band luminosity function with an extensive grid of synthetic cluster models obtained for a wide range of stellar ages, IMFs and star formation histories, suggests that the ages of these lower mass objects are at least few $10^{6}$ years.

The simultaneous presence of relatively old pre-main sequence objects with a massive YSO still in an active, pre-ZAMS, accretion phase, confirms that star formation tends to be an ongoing process for quite a long time span. The highest-mass star seems to be the last one to form.

The numerical importance of massive YSOs in a pre-ZAMS phase cannot be firmly established at the moment. Submillimeter surveys (Hill et al. 2005; Beltrán et al. 2006) reveal a consistent population of submillimeter cores in the proximity of IRAS point sources. These cores are devoid of mid-IR emission, but without an assessment of their far-infrared properties which only will allow firm temperature, luminosity and mass estimates, their nature will remain elusive. The PACS and SPIRE far-infrared cameras on board the Herschel satellite will be the ultimate tools to help distinguish between Hot-Cores, pre-ZAMS objects and quiescent cold cores. The recently approved Herschel KeyProject Hi-GAL for a complete 60-600 $\mu$ m continuum survey of the inner Galactic Plane will be invaluable to obtain firm statistics of massive YSOs in all evolutionary stages, thus providing a solid foundation to establish the timeline for the formation of intermediate- and high-mass stars.

\section{References}

Beltran, M. T., Brand, J., Cesaroni, R., et al. 2006, A\&A, 447, 221 Bessel, M. S. 1979, PASP, 91, 589

Beuther, H., Schilke, P., Sridharan, T. K., et al. 2002, ApJ 383, 892 Brand, J., \& Blitz, L. 1993, A\&A, 275, 67

Brand, J., Cesaroni, R., Palla, F., \& Molinari, S. 2001, A\&A, 370, 230

Fontani, F., Cesaroni, R., Testi, L., et al. 2004, A\&A, 414, 299

Habing, H. J. 1968, Bull. Astr. Inst. Netherlands, 19, 421

Hill, T., Burton, M. G., Minier, V., et al. 2005, MNRAS, 363, 405

Houck, J. R., Roellig, T. L., Van Cleve, J., et al. 2004, ApJS, 154, 18

Kaufman, M. J., Wolfire, M. G., \& Hollenbach, D. 2006, ApJ, 664, 283

Kennicutt, R. 1998, ApJ, 498, 541

McKee, C. F., \& Tan, J. C. 2003, ApJ, 585, 850

McKee, C. F., \& Williams, J. 1997, ApJ, 476, 144

Molinari, S. L., Brand, J., Cesaroni, R., \& Palla, F. 1996, A\&A, 308, 573

Molinari, S., Brand, J., Cesaroni, R., Palla, F., \& Palumbo, G. G. C. 1998a, A\&A, 336,339

Molinari, S., Testi, L., Brand, J., Cesaroni, R., \& Palla, F. 1998b, ApJ, 505, L39

Molinari, S., Brand, J., Cesaroni, R., \& Palla, F. 2000, A\&A, 355, 617

Molinari, S., Testi, L., Rodriguez, L. F., \& Zhang, Q. 2002, ApJ, 570, 758

Molinari, S., Pezzuto, S., Cesaroni, R., et al. 2008, A\&A, 481, 345

Morisset, C., Schaerer, D., Martin-Hernandez, N. L., et al. 2002, A\&A, 386, 558 Palla, F., Stahler, S. W. 1992, ApJ, 392, 667

Preibisch, Th., Ossenkopf, V., Yorke, H. W., \& Henning, Th. 1993, A\&A, 279, 577

Rieke, G. H., Young, E. T., Engelbracht, C. V., et al. 2004, ApJS, 154, 25

Robitaille, T. P., Whitney, B. A., Indebetouw, R., Wood, K., \& Denzmore, P. 2006, ApJS, 167, 256

Robitaille, T. P., Whitney, B. A., Indebetouw, R., \& Wood, K. 2007, ApJS, 169, 328

Shaver, P. A., McGee, R. X., Newton, L. M., Danks, A. C., \& Pottasch, S. R. 1983, MNRAS, 204, 53

Smith, J. D. T., Armus, L., Dale, D. A., et al. 2007, PASP, 119, 1133

Stecklum, B., Brandl, B., Henning, Th., et al. 2005, Poster presented at IAU227, Acireale, Catania, Italy, http://www.arcetri.astro.it/ iaus227/posters/stecklum_b.pdf

Thompson, R. I. 1984, ApJ, 283, 165

Thompson, M. A, \& Macdonald, G. H. 2003, A\&A, 407, 237

Tielens, A. G. G. M., \& Hollenbach, D. 1985, ApJ, 291, 722

Whitney, B. A., Wood, K., Bjorkman, J. E., \& Wolff, M. J. 2003, ApJ, 591, 1049

Zhang, Q., Hunter, T. R., Brand, J., et al. 2001, ApJ, 552, L167 